\title{
On the existence and uniqueness of solutions to stochastic 3-dimensional Lagrangian averaged Navier-Stokes equations $\dagger$
}

\author{
By Tomás Caraballo ${ }^{a}$, José Real ${ }^{a}$ and Takeshi Taniguchi ${ }^{b}$ \\ a Departamento de Ecuaciones Diferenciales y Análisis Numérico, \\ Universidad de Sevilla, Apdo. de Correos 1160, \\ 41080-Sevilla, Spain \\ ${ }^{b}$ Department of Mathematical Science, \\ Graduate School of Comparative Culture, \\ Kurume University, Miimachi, Kurume, \\ Fukuoka 839-5392, Japan
}

\begin{abstract}
We prove existence and uniqueness of solutions for a stochastic version of the 3DLagrangian averaged Navier-Stokes (LANS- $\alpha$ ) equation in a bounded domain. To this end, we previously prove some existence and uniqueness results for an abstract stochastic equation and justify that our model falls within this framework.
\end{abstract}

Keywords: 3D-Lagrangian averaged Navier-Stokes equations, variational solutions, cylindrical Wiener process

\section{Introduction}

Over the last decades, several turbulence models have been proposed for obtaining closure, i.e., for capturing the physical phenomenon of turbulence at computably low resolution. The Lagrangian-Averaged Navier-Stokes alpha (LANS- $\alpha$ ) model is the first to use Lagrangian averaging to address the turbulence closure problem, and one of the main reason justifying its use is the high computational cost that the Navier-Stokes model requires.

The LANS- $\alpha$ model provides closure by modifying the nonlinearity in the NavierStokes equations to stop the cascading of turbulence at scales smaller than a certain length, but without introducing any extra dissipation (see Holm et. al (2005) for a nice detailed description of the development of the LANS- $\alpha$ model).

It is well known that the relation of the Navier-Stokes equations to the phenomenon of turbulence have fascinated the physicists and mathematicians for a long time. One of the popular hypothesis relates the onset of turbulence to the randomness of background movement. Bensoussan \& Temam (1973) pioneered an analytic version of this approach based on investigations of stochastic Navier-Stokes

† E-mails: caraball@us.es (T. Caraballo); jreal@us.es (J. Real); takesi-taniguchi@mvc.biglobe.ne.jp (T. Taniguchi) 
equations driven by white noise type random forces, allowing to analyse a more realistic model for the problem, since it is sensible to consider some kind of 'noise' in the equations which may reflect, for instance, some environmental effects on the phenomenon, some external random forces, etc. Later, this approach was substantially developed and extended by many authors (see, e.g. Mikulevicius \& Rozovskii $(2004,2005)$ and the references therein). We are now proposing in this paper a stochastic version of the LANS- $\alpha$ model on a bounded domain which may be helpful and useful for future investigations towards a more complete knowledge and understanding of turbulence.

To be more precise, we study existence and uniqueness of solution for the 3D-Lagrangian Averaged Navier-Stokes (LANS- $\alpha$ ) equations, also called viscous Camassa-Holm equations by some authors (see Foias et al. (2002) and the references therein), with homogeneous Dirichlet boundary condition in a bounded domain, in the case in which random perturbations appear. More exactly, we suppose given a connected and bounded open subset $D$ of $\mathbb{R}^{3}$, with a Lipschitz boundary $\partial D$, and a final time $T>0$. We denote by $A$ the Stokes operator, and consider the system

$$
\left\{\begin{array}{l}
\partial_{t}(u-\alpha \Delta u)+\nu(A u-\alpha \Delta(A u))+(u \cdot \nabla)(u-\alpha \Delta u) \\
-\alpha \nabla u^{*} \cdot \Delta u+\nabla p=F(t, u)+G(t, u) \dot{W}_{t}, \quad \text { in } D \times(0, T), \\
\nabla \cdot u=0, \quad \text { in } D \times(0, T) \\
u=0, \quad A u=0, \quad \text { on } \partial D \times(0, T) \\
u(0)=u_{0}, \quad \text { in } D,
\end{array}\right.
$$

where $u=\left(u_{1}, u_{2}, u_{3}\right)$ and $p$ are unknown random fields on $D \times[0, T]$, representing, respectively, the large-scale (or averaged) velocity and the pressure, in each point of $D \times[0, T]$, of an incompressible viscous fluid with constant density filling the domain $D$. The constants $\nu>0$ and $\alpha>0$ are given, and represent respectively the kinematic viscosity of the fluid, and the square of the spatial scale at which fluid motion is filtered, $u_{0}$ is a given initial velocity field, and the terms $F(t, u)$ and $G(t, u) \dot{W}_{t}$ represent random external forces depending eventually on $u$, where $\dot{W}_{t}$ denotes the time derivative of a cylindrical Wiener process. The abstract and general form of this stochastic term allows to include in the formulation certain random environmental effects as well as the turbulent part of the velocity field, as happens in the case of Navier-Stokes equations (see Mikulevicius and Rozovskii (2004) for more details on the idea of splitting up the velocity field into a sum of slow oscillating-deterministic- and fast oscillating-stochastic-components).

To the best of our knowledge, this paper is the first work dealing with a stochastic version of the LANS- $\alpha$ model. In this sense, many topics and problems are to be solved. For instance, one first needs to choose an appropriate mathematical framework for setting up the problem, to prove existence, uniqueness and regularity of solutions, to analyse their long time behaviour, to investigate how close are the deterministic and stochastic versions when the intensity of the noise is small (this would justify whether or not the deterministic model is a good approximation of the more realistic stochastic one), to implement numerical simulations, to study the effects caused by the noise in the intermittency and anomalous scaling features of turbulence (see the nice description by Chertkov on this topics in page 136 of the paper by Ecke (2005)). To this respect, and taking into account that noise may have some regularizing and stabilising effects (as well as destabilising ones, see Caraballo 
\& Langa (2001) for more details) it is possible that, for suitable forms of the noisy term, one can obtain different results concerning these issues. However, with so many points to be addressed and treated, we will content ourselves in this paper with providing a suitable mathematical framework for (1.1) and prove existence and uniqueness of solution.

One of our main objectives is to show that the deterministic model used by Marsden and his collaborators (see, e.g., Marsden \& Shkoller (2001), Coutand \& Shkoller (2004) amongst others) on bounded domains is sensible in the case of bounded domains, in the sense that, when some stochastic disturbances appear or are taking into account in the model, we can propose a stochastic version which admits a rigorous mathematical treatment yielding to the existence and uniqueness of solutions of the problem (this is our first step in this investigation). In addition, our analysis also shows that the deterministic model is robust to stochastic perturbations of the kind considered. To do this, and instead of working directly with our LANS- $\alpha$ model, we first establish a result ensuring existence and uniqueness of solutions for a general model which contains this as a particular one, as well as the case of a periodic box (see Holm et al. (2002)), and other interesting situations from applications. As the proof strongly relies on the compactness of the injection between the spaces involved in the variational formulation, this technique is not suitable for more general unbounded domains. The analysis of the interesting situations related to infinite and semi-infinite domains (as channels, pipes, etc) will require of different techniques which are to be explored in the future.

We would like to mention briefly that there exists a controversy regarding the boundary condition $A u=0$. We only wish to point out here that, from the mathematical point of view, this condition makes sense in the case of bounded domains and contributes to the well-posedness of the problem (see Marsden \& Shkoller (2001), and Coutand et. al. (2003) for more details).

We hope that this initial work can serve as a motivating paper which can attract the attention and collaboration of researchers interested in this fascinating area of turbulence, and that their knowledge of the issues arisen in the deterministic framework, may serve as inspiration for obtaining as much understanding as possible of this stochastic model.

The content of the paper is as follows. In Section 2 we first establish and prove some properties of the nonlinear term appearing in our equations. The rigorous statement of our problem as well as the main results are included in Section 3. The existence and uniqueness of solutions for the abstract general equation are proved in Section 4. Finally, the proofs of our main results are given in the last section.

\section{Some results about the nonlinear term}

Previously to the formulation of our main results, we will obtain some results on the nonlinear term $(u \cdot \nabla)(u-\alpha \Delta u)-\alpha \nabla u^{*} \cdot \Delta u$ appearing in (1.1).

We will denote from now on by $(\cdot, \cdot)$ and $|\cdot|$, respectively, the scalar product and associated norm in $\left(L^{2}(D)\right)^{3}$, and by $(\nabla u, \nabla v)$ the scalar product in $\left(\left(L^{2}(D)\right)^{3}\right)^{3}$

of the gradients of $u$ and $v$. We consider the scalar product in $\left(H_{0}^{1}(D)\right)^{3}$ defined by $((u, v))=(u, v)+\alpha(\nabla u, \nabla v)$, for $u, v \in\left(H_{0}^{1}(D)\right)^{3}$, where its associated norm, which is in fact equivalent to the usual gradient norm, will be denoted by $\|\cdot\|$. Let us denote by $H$ the closure in $\left(L^{2}(D)\right)^{3}$ of the set $\mathcal{V}=\left\{v \in(\mathcal{D}(D))^{3}: \nabla \cdot v=0\right.$ in $\left.D\right\}$, 
and by $V$ the closure of $\mathcal{V}$ in $\left(H_{0}^{1}(D)\right)^{3}$. Then, $H$ is a Hilbert space equipped with the inner product of $\left(L^{2}(D)\right)^{3}$, and $V$ is a Hilbert subspace of $\left(H_{0}^{1}(D)\right)^{3}$.

We denote by $A$ the Stokes operator, with domain $D(A)=\left(H^{2}(D)\right)^{3} \cap V$, defined by $A w=-\mathcal{P}(\Delta w), \quad \forall w \in D(A)$, where $\mathcal{P}$ is the Leray operator, i.e., the projection operator from $\left(L^{2}(D)\right)^{3}$ onto $H$. Recall that as $\partial D$ is Lipschitz, $|A w|$ defines in $D(A)$ a norm which is equivalent to the $\left(H^{2}(D)\right)^{3}$-norm, i.e., there exists a constant $c_{1}>0$, depending only of $D$, such that

$$
\|w\|_{\left(H^{2}(D)\right)^{3}} \leq c_{1}|A w|, \quad \forall w \in D(A),
$$

and so $D(A)$ is a Hilbert space with the scalar product $(v, w)_{D(A)}=(A v, A w)$. For given $u \in D(A)$ and $v \in\left(L^{2}(D)\right)^{3}$, we define $(u \cdot \nabla) v$ as the element of $\left(H^{-1}(D)\right)^{3}$ given by

$$
\langle(u \cdot \nabla) v, w\rangle=\sum_{i, j=1}^{3}\left\langle\partial_{i} v_{j}, u_{i} w_{j}\right\rangle, \quad \forall w \in\left(H_{0}^{1}(D)\right)^{3} .
$$

Observe that (2.2) is meaningful, since $H^{2}(D) \subset L^{\infty}(D)$, and $H_{0}^{1}(D) \subset L^{6}(D)$, with continuous injections. This implies that $u_{i} w_{j} \in H_{0}^{1}(D)$, and there exists a constant $c_{2}>0$, depending only on $D$, such that

$$
|\langle(u \cdot \nabla) v, w\rangle| \leq c_{2}|A u||v|\|w\|, \forall(u, v, w) \in D(A) \times\left(L^{2}(D)\right)^{3} \times\left(H_{0}^{1}(D)\right)^{3} .
$$

Observe also that if $v \in\left(H^{1}(D)\right)^{3}$, then the definition above coincides with defining $(u \cdot \nabla) v$ as the vector function whose components are $\sum_{i=1}^{3} u_{i} \partial_{i} v_{j}$, for $j=1,2,3$.

Now, if $u \in D(A)$, then $\nabla u^{*} \in\left(H^{1}(D)\right)^{3 \times 3} \subset\left(L^{6}(D)\right)^{3 \times 3}$, and consequently, for $v \in\left(L^{2}(D)\right)^{3}$, we have that $\nabla u^{*} \cdot v \in\left(L^{3 / 2}(D)\right)^{3} \subset\left(H^{-1}(D)\right)^{3}$, with

$$
\left\langle\nabla u^{*} \cdot v, w\right\rangle=\sum_{i, j=1}^{3} \int_{D}\left(\partial_{j} u_{i}\right) v_{i} w_{j} d x, \quad \forall w \in\left(H_{0}^{1}(D)\right)^{3} .
$$

It is easy to see that there exists a constant $c_{3}>0$, depending only on $D$, such that

$$
\left|\left\langle\nabla u^{*} \cdot v, w\right\rangle\right| \leq c_{3}|A u\|v \mid\| w \|,
$$

for all $(u, v, w) \in D(A) \times\left(L^{2}(D)\right)^{3} \times\left(H_{0}^{1}(D)\right)^{3}$.

First, we have the following result.

Proposition 2.1. For all $(u, w) \in D(A) \times D(A)$ and all $v \in\left(L^{2}(D)\right)^{3}$, it holds

$$
\langle(u \cdot \nabla) v, w\rangle=-\left\langle\nabla w^{*} \cdot v, u\right\rangle .
$$

Proof. If $(u, w) \in D(A) \times D(A)$, then for each $i, j=1,2,3$, one has that $u_{i} w_{j} \in$ $H_{0}^{1}(D)$, and consequently,

$$
\left\langle\partial_{i} v_{j}, u_{i} w_{j}\right\rangle=-\int_{D} v_{j} \partial_{i}\left(u_{i} w_{j}\right) d x=-\int_{D} v_{j} w_{j} \partial_{i} u_{i} d x-\int_{D} v_{j} u_{i} \partial_{i} w_{j} d x .
$$

Thus, using that $\nabla \cdot u=0$, it is immediate to check that (2.6) is satisfied. 
We now consider the trilinear form defined by

$b^{\#}(u, v, w)=\langle(u \cdot \nabla) v, w\rangle+\left\langle\nabla u^{*} \cdot v, w\right\rangle, \quad \forall(u, v, w) \in D(A) \times\left(L^{2}(D)\right)^{3} \times\left(H_{0}^{1}(D)\right)^{3}$.

We then have the following result.

Proposition 2.2. The trilinear form $b^{\#}$ satisfies

$$
b^{\#}(u, v, w)=-b^{\#}(w, v, u), \quad \forall(u, v, w) \in D(A) \times\left(L^{2}(D)\right)^{3} \times D(A),
$$

and consequently,

$$
b^{\#}(u, v, u)=0, \quad \forall(u, v) \in D(A) \times\left(L^{2}(D)\right)^{3} .
$$

Moreover, there exists a constant $c>0$, depending only on $D$, such that

$$
\left|b^{\#}(u, v, w)\right| \leq c|A u||v||| w \|, \quad \forall(u, v, w) \in D(A) \times\left(L^{2}(D)\right)^{3} \times\left(H_{0}^{1}(D)\right)^{3},
$$

and

$$
\left|b^{\#}(u, v, w)\right| \leq c\|u\||v \|| A w \mid, \quad \forall(u, v, w) \in D(A) \times\left(L^{2}(D)\right)^{3} \times D(A) .
$$

Thus, in particular, $b^{\#}$ is continuous on $D(A) \times\left(L^{2}(D)\right)^{3} \times\left(H_{0}^{1}(D)\right)^{3}$.

Proof. The assertions are straightforward consequences of (2.6), (2.3) and (2.5).

\section{Statement of the problem and the main results}

Assume that $\{\Omega, \mathcal{F}, P\}$ is a complete probability space, and let $\left\{\mathcal{F}_{t}\right\}_{t \in[0, T]}$ be an increasing and right continuous family of sub $\sigma$-algebras of $\mathcal{F}$, such that $\mathcal{F}_{0}$ contains all the $P$-null sets of $\mathcal{F}$. Let $\left\{\beta_{t}^{j}, t \geq 0, j=1,2, \ldots\right\}$ be a given sequence of mutually independent standard real $\mathcal{F}_{t}$-Wiener processes defined on this space, and suppose given $K$, a separable Hilbert space, and $\left\{e_{j} ; j=1,2, \ldots\right\}$, an orthonormal basis of $K$. We denote by $\left\{W_{t} ; t \geq 0\right\}$, the cylindrical Wiener process with values in $K$ defined formally as $W_{t}=\sum_{j=1}^{\infty} \beta_{t}^{j} e_{j}$.

It is well known that this series does not converge in $K$, but rather in any Hilbert space $\widetilde{K}$ such that $K \subset \widetilde{K}$, being the injection of $K$ in $\widetilde{K}$ Hilbert-Schmidt (see e.g. DaPrato \& Zabczyk (1992) for more details).

For any separable Banach space $X$, and $p \in[1, \infty]$, we will denote by $M_{\mathcal{F}_{t}}^{p}(0, T ; X)$ the space of all processes $\varphi \in L^{p}(\Omega \times(0, T), d P \times d t ; X)$ that are $\mathcal{F}_{t}$-progressively measurable. The space $M_{\mathcal{F}_{t}}^{p}(0, T ; X)$ is a Banach subspace of $L^{p}(\Omega \times(0, T), d P \times$ $d t ; X)$.

We will write $L_{\mathcal{F}_{t}}^{p}(\Omega ; C([0, T] ; X))$, for $1 \leq p<\infty$, to denote the space of all continuous and $\mathcal{F}_{t}$-progressively measurable $X$-valued processes $\left\{\varphi_{t} ; 0 \leq t \leq T\right\}$ satisfying $E\left(\sup _{0 \leq t \leq T}\left\|\varphi_{t}\right\|_{X}^{p}\right)<\infty$.

Given another separable Hilbert space $\widetilde{H}$, with scalar product $(\cdot, \cdot)_{\widetilde{H}}$, let us denote by $\mathcal{L}^{2}(K ; \widetilde{H})$ the separable Hilbert space of Hilbert-Schmidt operators from $K$ into $\widetilde{H}$, and by $((\cdot, \cdot))_{\mathcal{L}^{2}(K ; \widetilde{H})}$ and $\|\cdot\|_{\mathcal{L}^{2}(K ; \widetilde{H})}$ the scalar product and its associated norm in $\mathcal{L}^{2}(K ; \widetilde{H})$.

For any process $\Psi \in M_{\mathcal{F}_{t}}^{2}\left(0, T ; \mathcal{L}^{2}(K ; \widetilde{H})\right)$, one can define the stochastic integral of 
$\Psi$ with respect to the cylindrical Wiener process $W_{t}$, denoted by $\int_{0}^{t} \Psi(s) d W_{s}, 0 \leq$ $t \leq T$, as the unique continuous $\widetilde{H}$-valued $\mathcal{F}_{t}$-martingale such that for all $h \in \widetilde{H}$, $\left(\int_{0}^{t} \Psi(s) d W_{s}, h\right)_{\widetilde{H}}=\sum_{j=1}^{\infty} \int_{0}^{t}\left(\Psi(s) e_{j}, h\right)_{\widetilde{H}} d \beta_{s}^{j}, \quad 0 \leq t \leq T$, where the integral with respect to $\beta_{s}^{j}$ is understood in the sense of Itô, and the series converges in $L^{2}(\Omega ; C([0, T]))$. See e.g. DaPrato \& Zabczyk (1992) for the properties of the stochastic integral so defined. In particular, we note that if $\Psi \in M_{\mathcal{F}_{t}}^{2}\left(0, T ; \mathcal{L}^{2}(K ; \widetilde{H})\right)$ and $\phi \in L^{2}\left(\Omega ; L^{\infty}(0, T ; \widetilde{H})\right)$ is $\mathcal{F}_{t}$-progressively measurable, then the series $\sum_{j=1}^{\infty} \int_{0}^{t}\left(\Psi(s) e_{j}, \phi(s)\right)_{\widetilde{H}} d \beta_{s}^{j}, \quad 0 \leq t \leq T$, converges in $L^{1}(\Omega ; C([0, T]))$, and defines a real valued continuous $\mathcal{F}_{t}$-martingale. We will use the notation $\int_{0}^{t}\left(\Psi(s), \phi(s) d W_{s}\right)$ $=\sum_{j=1}^{\infty} \int_{0}^{t}\left(\Psi(s) e_{j}, \phi(s)\right)_{\widetilde{H}} d \beta_{s}^{j}, \quad 0 \leq t \leq T$.

We suppose that $F$ and $G$ are measurable Lipschitz and sublinear mappings from $\Omega \times(0, T) \times V$ into $\left(H^{-1}(D)\right)^{3}$ and from $\Omega \times(0, T) \times V$ into $\mathcal{L}^{2}\left(K ;\left(L^{2}(D)\right)^{3}\right)$, respectively. More exactly, assume that, for all $v_{1}, v_{2} \in V, F\left(\cdot, v_{1}\right)$ and $G\left(\cdot, v_{1}\right)$ are $\mathcal{F}_{t}$-progressively measurable, and $d P \times d t$-a.e. in $\Omega \times(0, T)$,

$$
\begin{gathered}
\left\|F\left(t, v_{1}\right)-F\left(t, v_{2}\right)\right\|_{\left(H^{-1}(D)\right)^{3}} \leq L_{F}\left\|v_{1}-v_{2}\right\|, \\
F(t, 0) \in M_{\mathcal{F}_{t}}^{2}\left(0, T ;\left(H^{-1}(D)\right)^{3}\right), \\
\left\|G\left(t, v_{1}\right)-G\left(t, v_{2}\right)\right\|_{\mathcal{L}^{2}\left(K ;\left(L^{2}(D)\right)^{3}\right)} \leq L_{G}\left\|v_{1}-v_{2}\right\|, \\
G(t, 0) \in M_{\mathcal{F}_{t}}^{2}\left(0, T ; \mathcal{L}^{2}\left(K ;\left(L^{2}(D)\right)^{3}\right)\right) .
\end{gathered}
$$

Finally, we assume that

$$
u_{0} \in L^{2}\left(\Omega, \mathcal{F}_{0}, P ; V\right) .
$$

Definition 3.1. A variational solution to problem (1.1) is a stochastic process $u \in M_{\mathcal{F}_{t}}^{2}(0, T ; D(A)) \cap L^{2}\left(\Omega ; L^{\infty}(0, T ; V)\right)$, weakly continuous with values in $V$, such that for all $w \in D(A)$, and $t \in[0, T]$,

$$
\begin{aligned}
& ((u(t), w))+\nu \int_{0}^{t}(u(s)+\alpha A u(s), A w) d s \\
& +\int_{0}^{t} b^{\#}(u(s), u(s)-\alpha \Delta u(s), w) d s=\left(\left(u_{0}, w\right)\right) \\
& \quad+\int_{0}^{t}\langle F(s, u(s)), w\rangle d s+\left(\int_{0}^{t} G(s, u(s)) d W_{s}, w\right) .
\end{aligned}
$$

Our two major results are the following.

Proposition 3.2. Under the hypotheses (3.1), (3.2), (3.3), (3.4) and (3.5), there exists at most a variational solution of (1.1). Moreover, if $u$ is the variational solution of (1.1), then $u \in L^{2}(\Omega ; C([0, T] ; V))$ and satisfies

$$
\begin{aligned}
& \|u(t)\|^{2}+2 \nu \int_{0}^{t}(u(s)+\alpha A u(s), A u(s)) d s \\
& \leq\left\|u_{0}\right\|^{2}+2 \int_{0}^{t}\langle F(s, u(s)), u(s)\rangle d s \\
& \quad+2 \int_{0}^{t}\left(G(s, u(s)), u(s) d W_{s}\right)+\frac{1}{1+\alpha \mu_{1}} \int_{0}^{t} \| G\left(s, u(s) \|_{\mathcal{L}^{2}\left(K ;\left(L^{2}(D)\right)^{3}\right)}^{2} d s,\right.
\end{aligned}
$$


and $E \int_{0}^{t}\left(G(s, u(s)), u(s) d W_{s}\right)=0$, for all $t \in[0, T]$, where $\mu_{1}$ denotes the first eigenvalue of $A$.

Theorem 3.3. Suppose the hypotheses (3.1) and (3.3) hold, and that

$F(t, 0) \in L^{4}\left(\Omega ; L^{2}\left(0, T ;\left(H^{-1}(D)\right)^{3}\right)\right), \quad G(t, 0) \in L^{4}\left(\Omega ; L^{2}\left(0, T ; \mathcal{L}^{2}\left(K ;\left(L^{2}(D)\right)^{3}\right)\right)\right)$,

and $u_{0} \in L^{4}\left(\Omega, \mathcal{F}_{0}, P ; V\right)$. Then, there exists a unique variational solution $u$ of (1.1), and moreover, $u \in L^{4}(\Omega ; C([0, T] ; V)) \cap L^{4}\left(\Omega ; L^{2}(0, T ; D(A))\right)$. In fact, there exists $C>0$, depending only on $\alpha, \nu, T, L_{F}$ and $L_{G}$, such that

$$
\begin{aligned}
& E\left(\sup _{t \in[0, T]}\|u(t)\|^{4}\right)+E\left[\left(\int_{0}^{T}|A u(t)|^{2} d t\right)^{2}\right] \\
& \leq C\left[E\left(\left\|u_{0}\right\|^{4}\right)+E\left[\left(\int_{0}^{T}\|F(t, 0)\|_{\left(H^{-1}(D)\right)^{3}}^{2} d t\right)^{2}\right]+E\left[\left(\int_{0}^{T}\|G(t, 0)\|_{\mathcal{L}^{2}\left(K ;\left(L^{2}(D)\right)^{3}\right)}^{2} d t\right)^{2}\right]\right] .
\end{aligned}
$$

Moreover, associated to the variational solution $u$, there exists a unique $p \in L^{2}\left(\Omega, \mathcal{F}_{t}, P ; H^{-1}\left(0, t ; H^{-1}(D)\right)\right)$, for all $t \in(0, T]$, such that $P$-a.s.,

$$
\begin{aligned}
& \partial_{t}(u-\alpha \Delta u)+\nu(A u-\alpha \Delta(A u))+(u \cdot \nabla)(u-\alpha \Delta u) \\
& -\alpha \nabla u^{*} \cdot \Delta u+\nabla p=F(t, u)+G(t, u) \dot{W}_{t}, \text { in }\left(\mathcal{D}^{\prime}((0, T) \times D)\right)^{3}, \\
& \int_{D} p d x=0, \text { in } \mathcal{D}^{\prime}(0, T),
\end{aligned}
$$

where $G(t, u) \dot{W}_{t}$ denotes the time derivative of $\int_{0}^{t} G(s, u(s)) d W_{s}$, that is, by definition, we put

$$
G(t, u) \dot{W}_{t}=\partial_{t}\left(\int_{0}^{\cdot} G(s, u(s)) d W_{s}\right), \text { in } \mathcal{D}^{\prime}\left(0, T ;\left(L^{2}(D)\right)^{3}\right), P-\text { a.s.. }
$$

Although we could carry out a programme to prove these two results directly, we prefer to proceed in the following manner. We will establish in the next section some results concerning the existence and uniqueness of solutions for an abstract model. Then, we will be able to check that our situation falls within this framework, and consequently, our two main results will be automatically proved. In this way, we can obtain more profit from our analysis, since it may be possible that these abstract results can be applied to other situations arising in applications.

\section{Some abstract results}

Let $\mathcal{H}$ and $\mathcal{U}$ be two separable real Hilbert spaces, such that $\mathcal{U} \subset \mathcal{H}$ with compact injection, and $\mathcal{U}$ is dense in $\mathcal{H}$.

We denote by $(\cdot, \cdot)_{\mathcal{H}}$ and $((\cdot, \cdot))_{\mathcal{U}}$ the scalar product in $\mathcal{H}$ and $\mathcal{U}$ respectively, and we use $|\cdot|_{\mathcal{H}}$ and $\|\cdot\|_{\mathcal{U}}$ to denote their corresponding associated norms. We identify $\mathcal{H}$ with its topological dual $\mathcal{H}^{*}$, but we consider $\mathcal{U}$ as a subspace of $\mathcal{H}^{*}$.

We will denote by $\|\cdot\|_{\mathcal{U}^{*}}$ the norm in $\mathcal{U}^{*}$, and by $\langle\cdot, \cdot\rangle$ the duality product between $\mathcal{U}^{*}$ and $\mathcal{U}$. We then suppose given: 
a) An operator $\widetilde{A} \in \mathcal{L}\left(\mathcal{U}, \mathcal{U}^{*}\right)$, such that

a1) $\widetilde{A}$ is self adjoint,

a2) there exists $\widetilde{\alpha}>0$, such that

$$
2\langle\widetilde{A} v, v\rangle \geq \widetilde{\alpha}\|v\|_{\mathcal{U}}^{2}, \forall v \in \mathcal{U}
$$

Observe that there exist a Hilbert basis $\left\{v_{k} ; k \geq 1\right\} \subset \mathcal{U}$ of $\mathcal{H}$ and an increasing sequence $\left\{\lambda_{k} ; k \geq 1\right\} \subset(0, \infty)$ such that

$$
\widetilde{A} v_{k}=\lambda_{k} v_{k}, \quad \forall k \geq 1 .
$$

b) A bilinear mapping $\widetilde{B}: \mathcal{U} \times \mathcal{U} \rightarrow \mathcal{U}^{*}$ and a constant $\widetilde{c}>0$ such that

b1) $\langle\widetilde{B}(u, v), u\rangle=0$, for all $u, v \in \mathcal{U}$,

b2) $\|\widetilde{B}(u, v)\|_{\mathcal{U}^{*}} \leq \widetilde{c}|u|_{\mathcal{H}}\|v\|_{\mathcal{U}}$, for all $(u, v) \in \mathcal{U} \times \mathcal{U}$,

b3) $\langle\widetilde{B}(u, v), w\rangle \leq \widetilde{c}\|u\|_{\mathcal{U}}\|v\|_{\mathcal{U}}|w|_{\mathcal{H}}$, for all $u, v, w \in \mathcal{U}$.

c) A measurable random mapping $\widetilde{F}: \Omega \times(0, T) \times \mathcal{H} \rightarrow \mathcal{U}^{*}$, such that for fixed $h \in \mathcal{H}, \widetilde{F}(\cdot, h)$ is $\mathcal{F}_{t}$-progressively measurable,

c1) $\widetilde{F}(\cdot, 0) \in M_{\mathcal{F}_{t}}^{2}\left(0, T ; \mathcal{U}^{*}\right)$,

c2) there exists $L_{\widetilde{F}}>0$ such that $\|\widetilde{F}(t, u)-\widetilde{F}(t, v)\|_{\mathcal{U}^{*}} \leq L_{\widetilde{F}}|u-v|_{\mathcal{H}}, d P \times$ $d t-$ a.e., for all $u, v \in \mathcal{H}$.

d) A measurable random mapping $\widetilde{G}: \Omega \times(0, T) \times \mathcal{H} \rightarrow \mathcal{L}^{2}(K ; \mathcal{H})$, such that for fixed $h \in \mathcal{H}, \widetilde{G}(\cdot, h)$ is $\mathcal{F}_{t}$-progressively measurable,

d1) $\widetilde{G}(\cdot, 0) \in M_{\mathcal{F}_{t}}^{2}\left(0, T ; \mathcal{L}^{2}(K ; \mathcal{H})\right)$,

d2) there exists $L_{\widetilde{G}}>0$ such that $\|\widetilde{G}(t, u)-\widetilde{G}(t, v)\|_{\mathcal{L}^{2}(K ; \mathcal{H})} \leq L_{\widetilde{G}}|u-v|_{\mathcal{H}}$, $d P \times d t-$ a.e., for all $u, v \in \mathcal{H}$.

e) An initial datum $u_{0} \in L^{2}\left(\Omega, \mathcal{F}_{0}, P ; \mathcal{H}\right)$.

We consider the equation

$$
\begin{aligned}
& u(t)+\int_{0}^{t} \widetilde{A} u(s) d s+\int_{0}^{t} \widetilde{B}(u(s), u(s)) d s \\
& =u_{0}+\int_{0}^{t} \widetilde{F}(s, u(s)) d s+\int_{0}^{t} \widetilde{G}(s, u(s)) d W_{s}, P-\text { a.s., } \forall t \in[0, T] .
\end{aligned}
$$

Definition 4.1. A solution of $(4.3)$ is a process $u \in M_{\mathcal{F}_{t}}^{2}(0, T ; \mathcal{U}) \cap L^{2}\left(\Omega ; L^{\infty}(0, T ; \mathcal{H})\right)$, such that the equation (4.3) is satisfied in $\mathcal{U}^{*}, P-$ a.s. for all $t \in[0, T]$.

Proposition 4.2. If $u$ is a solution of (4.3), then $u \in L^{2}(\Omega ; C([0, T] ; \mathcal{H}))$, and

$$
\begin{gathered}
|u(t)|_{\mathcal{H}}^{2}+2 \int_{0}^{t}\langle\widetilde{A} u(s), u(s)\rangle d s=\left|u_{0}\right|_{\mathcal{H}}^{2}+2 \int_{0}^{t}\langle\widetilde{F}(s, u(s)), u(s)\rangle d s \\
\quad+2 \int_{0}^{t}\left(\widetilde{G}(s, u(s)), u(s) d W_{s}\right)+\int_{0}^{t}\|\widetilde{G}(s, u(s))\|_{\mathcal{L}^{2}(K ; \mathcal{H})}^{2}, \forall t \in[0, T],
\end{gathered}
$$

and

$$
E \int_{0}^{t}\left(\widetilde{G}(s, u(s)), u(s) d W_{s}\right)=0, \forall t \in[0, T] .
$$


Proof. If $u$ is a solution of (4.3), then $\widetilde{B}(u, u) \in M_{\mathcal{F}_{t}}^{1}\left(0, T ; \mathcal{U}^{*}\right), \widetilde{A} u-\widetilde{F}(t, u) \in$ $M_{\mathcal{F}_{t}}^{2}\left(0, T ; \mathcal{U}^{*}\right)$, and $\widetilde{G}(t, u) \in M_{\mathcal{F}_{t}}^{2}\left(0, T ; \mathcal{L}^{2}(K ; \mathcal{H})\right)$. Consequently, from Theorem 3.2 in Pardoux (1975) (page 58), $u$ is $P$-a.s. continuous with values in $\mathcal{H}$, and by b1), the energy equality (4.4) is satisfied. Finally, (4.5) is a direct consequence of the fact that $\widetilde{G}(t, u) \in M_{\mathcal{F}_{t}}^{2}\left(0, T ; \mathcal{L}^{2}(K ; \mathcal{H})\right)$ and $u \in L^{2}\left(\Omega ; L^{\infty}(0, T ; \mathcal{H})\right)$.

Proposition 4.3. There exists at most one solution of (4.3).

Proof. Let $u^{1}$ and $u^{2}$ be two solutions of (4.3), and denote $\bar{u}=u^{1}-u^{2}$. Then, reasoning as in the proof of Proposition 4.2, and taking into account b1), we obtain

$$
\begin{aligned}
|\bar{u}(t)|_{\mathcal{H}}^{2} & +2 \int_{0}^{t}\langle\widetilde{A} \bar{u}(s), \bar{u}(s)\rangle d s+2 \int_{0}^{t}\left\langle\widetilde{B}\left(u^{2}(s), \bar{u}(s)\right), \bar{u}(s)\right\rangle d s \\
& =2 \int_{0}^{t}\left\langle\widetilde{F}\left(s, u^{1}(s)\right)-\widetilde{F}\left(s, u^{2}(s)\right), \bar{u}(s)\right\rangle d s \\
& +\int_{0}^{t}\left\|\widetilde{G}\left(s, u^{1}(s)\right)-\widetilde{G}\left(s, u^{2}(s)\right)\right\|_{\mathcal{L}^{2}(K ; \mathcal{H})}^{2} d s \\
& +2 \int_{0}^{t}\left(\widetilde{G}\left(s, u^{1}(s)\right)-\widetilde{G}\left(s, u^{2}(s)\right), \bar{u}(s) d W_{s}\right), \quad \forall t \in[0, T] .
\end{aligned}
$$

Take $\mu>0$ to be fixed later and define $\sigma(t)=\exp \left(-\mu \int_{0}^{t}\left\|u^{2}(s)\right\|_{\mathcal{U}}^{2} d s\right), 0 \leq t \leq T$. Applying Itô's formula to the real process $\sigma(t)|\bar{u}(t)|_{\mathcal{H}}^{2}$, we obtain from (4.6), a2), b3), c2) and d2), that

$$
\begin{aligned}
& \sigma(t)|\bar{u}(t)|_{\mathcal{H}}^{2}+\widetilde{\alpha} \int_{0}^{t} \sigma(s)\|\bar{u}(s)\|_{\mathcal{U}}^{2} d s \leq L_{\widetilde{G}}^{2} \int_{0}^{t} \sigma(s)|\bar{u}(s)|_{\mathcal{H}}^{2} d s \\
& +2 \widetilde{c} \int_{0}^{t} \sigma(s)\left\|u^{2}(s)\right\|_{\mathcal{U}}\|\bar{u}(s)\|_{\mathcal{U}}|\bar{u}(s)|_{\mathcal{H}} d s+2 L_{\widetilde{F}} \int_{0}^{t} \sigma(s)\|\bar{u}(s)\|_{\mathcal{U}}|\bar{u}(s)|_{\mathcal{H}} d s \\
& +2 \int_{0}^{t}\left(\sigma(s)\left(\widetilde{G}\left(s, u^{1}(s)\right)-\widetilde{G}\left(s, u^{2}(s)\right)\right), \bar{u}(s) d W_{s}\right) \\
& -\mu \int_{0}^{t} \sigma(s)\left\|u^{2}(s)\right\|_{\mathcal{U}}^{2}|\bar{u}(s)|_{\mathcal{H}}^{2} d s, \quad \forall t \in[0, T]
\end{aligned}
$$

But,

$$
2 \widetilde{c}\left\|u^{2}(s)\right\|_{\mathcal{U}}\|\bar{u}(s)\|_{\mathcal{U}}|\bar{u}(s)|_{\mathcal{H}} \leq \frac{\widetilde{\alpha}}{2}\|\bar{u}(s)\|_{\mathcal{U}}^{2}+\frac{2 \widetilde{c}^{2}}{\widetilde{\alpha}}\left\|u^{2}(s)\right\|_{\mathcal{U}}^{2}|\bar{u}(s)|_{\mathcal{H}}^{2}
$$

and

$$
2 L_{\widetilde{F}}\|\bar{u}(s)\|_{\mathcal{U}}|\bar{u}(s)|_{\mathcal{H}} \leq \frac{\widetilde{\alpha}}{2}\|\bar{u}(s)\|_{\mathcal{U}}^{2}+\frac{2 L_{\widetilde{F}}^{2}}{\widetilde{\alpha}}|\bar{u}(s)|_{\mathcal{H}}^{2} .
$$

If we take $\mu=2 \widetilde{c}^{2} / \widetilde{\alpha}$, we obtain from (4.7),

$$
\begin{aligned}
\sigma(t)|\bar{u}(t)|_{\mathcal{H}}^{2} \leq\left(L_{\widetilde{G}}^{2}+\frac{2 L_{\widetilde{F}}^{2}}{\widetilde{\alpha}}\right) \int_{0}^{t} \sigma(s)|\bar{u}(s)|_{\mathcal{H}}^{2} d s \\
\quad+2 \int_{0}^{t}\left(\sigma(s)\left(\widetilde{G}\left(s, u^{1}(s)\right)-\widetilde{G}\left(s, u^{2}(s)\right)\right), \bar{u}(s) d W_{s}\right), \quad \forall t \in[0, T] .
\end{aligned}
$$


As $0<\sigma(t) \leq 1$, the expectation of the stochastic integral in (4.8) vanishes, and

$$
E \sigma(t)|\bar{u}(t)|_{\mathcal{H}}^{2} \leq\left(L_{\widetilde{G}}^{2}+\frac{2 L_{\widetilde{F}}^{2}}{\widetilde{\alpha}}\right) E \int_{0}^{t} \sigma(s)|\bar{u}(s)|_{\mathcal{H}}^{2} d s, 0 \leq t \leq T .
$$

The Gronwall Lemma implies now that $\bar{u}(t)=0, P$-a.s. for all $t \in[0, T]$.

Now, we can prove the following result.

Theorem 4.4. Suppose all the above hypotheses and that, moreover, $\widetilde{F}(\cdot, 0) \in$ $L^{4}\left(\Omega ; L^{2}\left(0, T ; \mathcal{U}^{*}\right)\right), \widetilde{G}(\cdot, 0) \in L^{4}\left(\Omega ; L^{2}\left(0, T ; \mathcal{L}^{2}(K ; \mathcal{H})\right)\right)$ and $u_{0} \in L^{4}\left(\Omega, \mathcal{F}_{0}, P ; \mathcal{H}\right)$. Then, there exists a unique solution u to (4.3), which satisfies in addition,

$$
u \in L^{4}(\Omega ; C([0, T] ; \mathcal{H})) \cap L^{4}\left(\Omega ; L^{2}(0, T ; \mathcal{U})\right) .
$$

In fact, there exists $C>0$, depending only on $\widetilde{\alpha}, T, L_{\widetilde{F}}$ and $L_{\widetilde{G}}$ such that

$$
\begin{array}{r}
E\left(\sup _{[0, T]}|u(t)|_{\mathcal{H}}^{4}\right)+E\left[\left(\int_{0}^{T}\|u(t)\|_{\mathcal{U}}^{2} d t\right)^{2}\right] \\
\leq C\left[E\left(\left|u_{0}\right|_{\mathcal{H}}^{4}\right)+E\left[\left(\int_{0}^{T}\|\widetilde{F}(t, 0)\|_{\mathcal{U}^{*}}^{2} d t\right)^{2}\right]\right. \\
\left.+E\left[\left(\int_{0}^{T}\|\widetilde{G}(t, 0)\|_{\mathcal{L}^{2}(K ; \mathcal{H})}^{2} d t\right)^{2}\right]\right] .
\end{array}
$$

Proof. The proof follows the scheme of that in Breckner (1999) for the case of stochastic 2D-Navier-Stokes equations, but with appropriate changes (see also Bensoussan 1995). We will split the proof into five steps.

Step 1.- Construction of an approximating sequence.

We take the Hilbert basis $\left\{v_{k} ; k \geq 1\right\} \subset \mathcal{U}$ of $\mathcal{H}$ satisfying (4.2). For each integer $m \geq 1$, we denote by $\mathcal{H}_{m}=\mathcal{U}_{m}$ the vector space spanned by $\left\{v_{1}, \ldots, v_{m}\right\}$, and consider the finite dimensional problem

$$
\begin{aligned}
\left(u_{m}(t), v_{k}\right)_{\mathcal{H}}+\int_{0}^{t}\left\langle\widetilde{A} u_{m}(s), v_{k}\right\rangle d s+\int_{0}^{t}\left\langle\widetilde{B}\left(u_{m}(s), u_{m}(s)\right), v_{k}\right\rangle d s \\
=\left(u_{0}, v_{k}\right)_{\mathcal{H}}+\int_{0}^{t}\left\langle\widetilde{F}\left(s, u_{m}(s)\right), v_{k}\right\rangle d s \\
\quad+\sum_{j=1}^{m} \int_{0}^{t}\left(\widetilde{G}\left(s, u_{m}(s)\right) e_{j}, v_{k}\right)_{\mathcal{H}} d \beta_{s}^{j}, 0 \leq t \leq T, 1 \leq k \leq m .
\end{aligned}
$$

Arguing as in the proof of Theorem 1.2.1 in Breckner (1999), pages 11-13, one can obtain existence and uniqueness of a solution $u_{m} \in M_{\mathcal{F}_{t}}^{2}\left(0, T ; \mathcal{U}_{m}\right)$ of equation (4.10) with continuous trajectories. 
Step 2.- Estimates for the approximating sequence.

By Itô's formula and b1), we obtain for all $t \in[0, T]$

$$
\begin{aligned}
& \left|u_{m}(t)\right|_{\mathcal{H}}^{2}+2 \int_{0}^{t}\left\langle\widetilde{A} u_{m}(s), u_{m}(s)\right\rangle d s \\
& =\sum_{k=1}^{m}\left(u_{0}, v_{k}\right)_{\mathcal{H}}^{2}+2 \int_{0}^{t}\left\langle\widetilde{F}\left(s, u_{m}(s)\right), u_{m}(s)\right\rangle d s \\
& +\int_{0}^{t} \sum_{j, k=1}^{m}\left(\widetilde{G}\left(s, u_{m}(s) e_{j}, v_{k}\right)_{\mathcal{H}}^{2} d s+2 \sum_{j=1}^{m} \int_{0}^{t}\left(\widetilde{G}\left(s, u_{m}(s)\right) e_{j}, u_{m}(s)\right)_{\mathcal{H}} d \beta_{s}^{j} .\right.
\end{aligned}
$$

But then, taking into account that $\sum_{j, k=1}^{m}\left(\widetilde{G}\left(s, u_{m}(s)\right) e_{j}, v_{k}\right)_{\mathcal{H}}^{2} \leq\left\|\widetilde{G}\left(s, u_{m}(s)\right)\right\|_{\mathcal{L}^{2}(K ; \mathcal{H})}^{2}$, the fact that

$$
\begin{aligned}
& 2\left\langle\widetilde{F}\left(s, u_{m}(s)\right), u_{m}(s)\right\rangle \leq 2 L_{\widetilde{F}}\left|u_{m}(s)\right|_{\mathcal{H}}\left\|u_{m}(s)\right\|_{\mathcal{U}}+2\|\widetilde{F}(s, 0)\|_{\mathcal{U}^{*}}\left\|u_{m}(s)\right\|_{\mathcal{U}} \\
& \leq \frac{\widetilde{\alpha}}{2}\left\|u_{m}(s)\right\|_{\mathcal{U}}^{2}+\frac{4 L_{\mathcal{F}}^{2}}{\widetilde{\alpha}}\left|u_{m}(s)\right|_{\mathcal{H}}^{2}+\frac{4}{\widetilde{\alpha}}\|\widetilde{F}(s, 0)\|_{\mathcal{U}^{*}}^{2},
\end{aligned}
$$

and a2), d1) and d2), we deduce from (4.11) that

$$
\begin{aligned}
& \left|u_{m}(t)\right|_{\mathcal{H}}^{2}+\frac{\widetilde{\alpha}}{2} \int_{0}^{t}\left\|u_{m}(s)\right\|_{\mathcal{U}}^{2} d s \\
& \leq\left|u_{0}\right|_{\mathcal{H}}^{2}+\left(\frac{4 L_{\widetilde{F}}^{2}}{\widetilde{\alpha}}+2 L_{\widetilde{G}}^{2}\right) \int_{0}^{t}\left|u_{m}(s)\right|_{\mathcal{H}}^{2} d s \\
& +\frac{4}{\widetilde{\alpha}} \int_{0}^{t}\|\widetilde{F}(s, 0)\|_{\mathcal{U}^{*}}^{2} d s+2 \int_{0}^{t}\|\widetilde{G}(s, 0)\|_{\mathcal{L}^{2}(K ; \mathcal{H})}^{2} d s \\
& +2 \sum_{j=1}^{m} \int_{0}^{t}\left(\widetilde{G}\left(s, u_{m}(s) e_{j}, u_{m}(s)\right)_{\mathcal{H}} d \beta_{s}^{j}, \quad \forall t \in[0, T] .\right.
\end{aligned}
$$

For each integer $n \geq 1$, consider the $\mathcal{F}_{t}$-stopping time $\tau_{n}^{m}$ defined by

$$
\tau_{n}^{m}=\min \left(T, \inf \left\{t \in[0, T] ;\left|u_{m}(t)\right|_{\mathcal{H}}^{2}+\int_{0}^{t}\left\|u_{m}(s)\right\|_{\mathcal{U}}^{2} d s \geq n^{2}\right\}\right)
$$

For fixed $m$, the sequence $\left\{\tau_{n}^{m} ; n \geq 1\right\}$ is increasing to $T$. It follows from (4.12),

$$
\begin{aligned}
\sup _{s \in\left[0, t \wedge \tau_{n}^{m}\right]}\left|u_{m}(s)\right|_{\mathcal{H}}^{4}+\frac{\widetilde{\alpha}^{2}}{4}\left(\int_{0}^{t \wedge \tau_{n}^{m}}\left\|u_{m}(s)\right\|_{\mathcal{U}}^{2} d s\right)^{2} \\
\leq 6\left(\left|u_{0}\right|_{\mathcal{H}}^{2}+\frac{4}{\widetilde{\alpha}} \int_{0}^{T}\|\widetilde{F}(s, 0)\|_{\mathcal{U}^{*}}^{2} d s+2 \int_{0}^{T}\|\widetilde{G}(s, 0)\|_{\mathcal{L}^{2}(K ; \mathcal{H})}^{2} d s\right)^{2} \\
\quad+6 T\left(\frac{4 L_{\widetilde{F}}^{2}}{\widetilde{\alpha}}+2 L_{\widetilde{G}}^{2}\right)^{2} \int_{0}^{t} \sup _{r \in\left[0, s \wedge \tau_{n}^{m}\right]}\left|u_{m}(r)\right|_{\mathcal{H}}^{4} d s \\
\quad+24 \sup _{s \in\left[0, t \wedge \tau_{n}^{m}\right]}\left(\sum_{j=1}^{m} \int_{0}^{s}\left(\widetilde{G}\left(r, u_{m}(r) e_{j}, u_{m}(r)\right)_{\mathcal{H}} d \beta_{r}^{j}\right)^{2}\right.
\end{aligned}
$$

Article submitted to Royal Society 
for all $t \in[0, T]$ and all $m, n \geq 1$.

But, taking expectations in (4.13), observing that by Doob's inequality it holds

$$
\begin{aligned}
E \sup _{s \in\left[0, t \wedge \tau_{n}^{m}\right]}\left(\sum_{j=1}^{m} \int_{0}^{s}\left(\widetilde{G}\left(r, u_{m}(r) e_{j}, u_{m}(r)\right)_{\mathcal{H}} d \beta_{r}^{j}\right)^{2}\right. \\
\leq 4 E \sum_{j=1}^{m} \int_{0}^{t \wedge \tau_{n}^{m}}\left(\widetilde{G}\left(s, u_{m}(s) e_{j}, u_{m}(s)\right)_{\mathcal{H}}^{2} d s\right. \\
\leq 4 E\left(\sup _{s \in\left[0, t \wedge \tau_{n}^{m}\right]}\left|u_{m}(s)\right|_{\mathcal{H}}^{2} \int_{0}^{t \wedge \tau_{n}^{m}}\left\|\widetilde{G}\left(s, u_{m}(s)\right)\right\|_{\mathcal{L}^{2}(K ; \mathcal{H})}^{2} d s\right) \\
\leq \frac{1}{2} E \sup _{s \in\left[0, t \wedge \tau_{n}^{m}\right]}\left|u_{m}(t)\right|_{\mathcal{H}^{\prime}}^{4}+64 T L_{\widetilde{G}}^{4} E \int_{0}^{t \wedge \tau_{n}^{m}}\left|u_{m}(s)\right|_{\mathcal{H}}^{4} d s \\
\quad+64 E\left[\left(\int_{0}^{T}\|\widetilde{G}(s, 0)\|_{\mathcal{L}^{2}(K ; \mathcal{H})}^{2} d s\right)^{2}\right],
\end{aligned}
$$

and, using the Gronwall Lemma and the fact that $\tau_{n}^{m} \uparrow T$ when $n$ goes to $\infty$, it follows that there exists a constant $C_{1}>0$, depending only on $\widetilde{\alpha}, T, L_{\widetilde{F}}$ and $L_{\widetilde{G}}$, such that, for all $m \geq 1$,

$$
\begin{aligned}
& E\left(\sup _{[0, T]}\left|u_{m}(t)\right|_{\mathcal{H}}^{4}\right)+E\left[\left(\int_{0}^{T}\left\|u_{m}(t)\right\|_{\mathcal{U}}^{2} d t\right)^{2}\right] \\
& \leq C_{1}\left[E\left(\left|u_{0}\right|_{\mathcal{H}}^{4}\right)+E\left[\left(\int_{0}^{T}\|\widetilde{F}(t, 0)\|_{\mathcal{U}^{*}}^{2} d t\right)^{2}\right]+E\left[\left(\int_{0}^{T}\|\widetilde{G}(t, 0)\|_{\mathcal{L}^{2}(K ; \mathcal{H})}^{2} d t\right)^{2}\right]\right]
\end{aligned}
$$

Now, observing that

$$
\int_{0}^{T}\left|u_{m}(s)\right|_{\mathcal{H}}^{2}\left\|u_{m}(s)\right\|_{\mathcal{U}}^{2} d s \leq \sup _{t \in[0, T]}\left|u_{m}(t)\right|_{\mathcal{H}}^{2} \int_{0}^{T}\left\|u_{m}(t)\right\|_{\mathcal{U}}^{2} d t,
$$

one can obtain from (4.15) and for all $m \geq 1$,

$$
\begin{aligned}
E \int_{0}^{T}\left|u_{m}(s)\right|_{\mathcal{H}}^{2}\left\|u_{m}(s)\right\|_{\mathcal{U}}^{2} d s \leq & \frac{C_{1}}{2}\left[E\left(\left|u_{0}\right|_{\mathcal{H}}^{4}\right)+E\left[\left(\int_{0}^{T}\|\widetilde{F}(t, 0)\|_{\mathcal{U}^{*}}^{2} d t\right)^{2}\right]\right. \\
+E & {\left.\left[\left(\int_{0}^{T}\|\widetilde{G}(t, 0)\|_{\mathcal{L}^{2}(K ; \mathcal{H})}^{2} d t\right)^{2}\right]\right] }
\end{aligned}
$$

Step 3.- Taking limits in the finite dimensional equations.

From b2) and (4.16), we can deduce that the sequence $\widetilde{B}\left(u_{m}, u_{m}\right)$ is bounded in $M_{\mathcal{F}_{t}}^{2}\left(0, T ; \mathcal{U}^{*}\right)$. On the other hand, from $\left.(4.15), \mathrm{c} 2\right)$ and $\left.\mathrm{d} 2\right)$, we have that, in particular, the sequence $u_{m}$ is bounded in $M_{\mathcal{F}_{t}}^{2}(0, T ; \mathcal{U})$, the sequence $u_{m}(0)$ is bounded in 
$L^{4}\left(\Omega, \mathcal{F}_{0}, P ; \mathcal{H}\right)$, the sequence $\widetilde{F}\left(t, u_{m}\right)$ is bounded in $M_{\mathcal{F}_{t}}^{2}\left(0, T ; \mathcal{U}^{*}\right)$, and $\widetilde{G}\left(t, u_{m}\right)$ is bounded in $M_{\mathcal{F}_{t}}^{2}\left(0, T ; \mathcal{L}^{2}(K ; \mathcal{H})\right)$.

Thus, we can ensure that there exists a subsequence $\left\{u_{m^{\prime}}\right\} \subset\left\{u_{m}\right\}$, and five elements $u \in M_{\mathcal{F}_{t}}^{2}(0, T ; \mathcal{U}), \xi \in L^{4}\left(\Omega, \mathcal{F}_{0}, P ; \mathcal{H}\right), \mathcal{B} \in M_{\mathcal{F}_{t}}^{2}\left(0, T ; \mathcal{U}^{*}\right), \Phi \in$ $M_{\mathcal{F}_{t}}^{2}\left(0, T ; \mathcal{U}^{*}\right)$, and $\mathcal{G} \in M_{\mathcal{F}_{t}}^{2}\left(0, T ; \mathcal{L}^{2}(K ; \mathcal{H})\right)$, such that

$$
\begin{gathered}
u_{m^{\prime}} \rightarrow u \text { in } M_{\mathcal{F}_{t}}^{2}(0, T ; \mathcal{U}), \\
u_{m^{\prime}}(0) \rightarrow \xi \text { in } L^{4}\left(\Omega, \mathcal{F}_{0}, P ; \mathcal{H}\right), \\
\widetilde{B}\left(u_{m^{\prime}}, u_{m^{\prime}}\right) \rightarrow \mathcal{B} \text { in } M_{\mathcal{F}_{t}}^{2}\left(0, T ; \mathcal{U}^{*}\right), \\
\widetilde{F}\left(t, u_{m^{\prime}}\right) \rightarrow \Phi \text { in } M_{\mathcal{F}_{t}}^{2}\left(0, T ; \mathcal{U}^{*}\right), \\
\widetilde{G}\left(t, u_{m^{\prime}}\right) \rightarrow \mathcal{G} \text { in } M_{\mathcal{F}_{t}}^{2}\left(0, T ; \mathcal{L}^{2}(K ; \mathcal{H})\right) .
\end{gathered}
$$

It is then a standard matter (see e.g. Pardoux 1975) to obtain from (4.10) that $u \in L^{2}(\Omega ; C([0, T] ; \mathcal{H}))$ and satisfies for all $0 \leq t \leq T$ that

$$
u(t)+\int_{0}^{t} \widetilde{A} u(s) d s+\int_{0}^{t} \mathcal{B}(s) d s=u_{0}+\int_{0}^{t} \Phi(s) d s+\int_{0}^{t} \mathcal{G}(s) d W_{s},
$$

Step 4.- To prove that $\mathcal{B}=\widetilde{B}(u, u), \Phi=\widetilde{F}(t, u)$ and $\mathcal{G}=\widetilde{G}(t, u)$.

For simplicity we will keep denoting by $\left\{u_{m}\right\}$ the subsequence $\left\{u_{m^{\prime}}\right\}$ in this step. For each $m \geq 1$, let us denote $\widetilde{u}_{m}(t)=\Pi_{m} u(t)=\sum_{k=1}^{m}\left(u(t), v_{k}\right)_{\mathcal{H}} v_{k}$, where $\Pi_{m} \in \mathcal{L}\left(\mathcal{H}, \mathcal{H}_{m}\right)$ is the orthogonal projection of $\mathcal{H}$ onto $\mathcal{H}_{m}$. It follows that

$$
\left|\widetilde{u}_{m}(t)\right|_{\mathcal{H}} \leq|u(t)|_{\mathcal{H}}, \quad\left\|\widetilde{u}_{m}(t)\right\|_{\mathcal{U}} \leq \mu\|u(t)\|_{\mathcal{U}},
$$

with $\mu=(2\|\widetilde{A}\| / \widetilde{\alpha})^{1 / 2}$, and

$$
\widetilde{u}_{m} \rightarrow u \text { in } M_{\mathcal{F}_{t}}^{2}(0, T ; \mathcal{U}) .
$$

On the other hand, we obviously have $\left(\widetilde{u}_{m}(t), v_{k}\right)_{\mathcal{H}}=\left(u(t), v_{k}\right)_{\mathcal{H}}, \forall 1 \leq k \leq m$. And also, by a1) and (4.2), we easily obtain for each $1 \leq k \leq m$, that $\left\langle\widetilde{A} \widetilde{u}_{m}(t), v_{k}\right\rangle=$ $\left\langle\widetilde{A} u(t), v_{k}\right\rangle$.

From (4.22) and (4.10), it follows for all $t \in[0, T]$, all $1 \leq k \leq m$ and all $m \geq 1$,

$$
\begin{aligned}
& \left(\widetilde{u}_{m}(t)-u_{m}(t), v_{k}\right)_{\mathcal{H}}+\int_{0}^{t}\left\langle\widetilde{A}\left(\widetilde{u}_{m}(s)-u_{m}(s)\right), v_{k}\right\rangle d s \\
& +\int_{0}^{t}\left\langle\mathcal{B}(s)-\widetilde{B}\left(u_{m}(s), u_{m}(s)\right), v_{k}\right\rangle d s=\int_{0}^{t}\left\langle\Phi(s)-\widetilde{F}\left(s, u_{m}(s)\right), v_{k}\right\rangle d s \\
& +\sum_{j=1}^{m} \int_{0}^{t}\left(\mathcal{G}(s) e_{j}-\widetilde{G}\left(s, u_{m}(s)\right) e_{j}, v_{k}\right)_{\mathcal{H}} d \beta_{s}^{j}+\sum_{j=m+1}^{\infty} \int_{0}^{t}\left(\mathcal{G}(s) e_{j}, v_{k}\right)_{\mathcal{H}} d \beta_{s}^{j}
\end{aligned}
$$

Thus, by Itô's formula,

$$
\begin{aligned}
& d\left[\left(\widetilde{u}_{m}(t)-u_{m}(t), v_{k}\right)_{\mathcal{H}}^{2}\right]=2\left(\widetilde{u}_{m}(t)-u_{m}(t), v_{k}\right)_{\mathcal{H}} d\left[\left(\widetilde{u}_{m}(t)-u_{m}(t), v_{k}\right)_{\mathcal{H}}\right] \\
& +\sum_{j=1}^{m}\left(\mathcal{G}(t) e_{j}-\widetilde{G}\left(t, u_{m}(t)\right) e_{j}, v_{k}\right)_{\mathcal{H}}^{2} d t+\sum_{j=m+1}^{\infty}\left(\mathcal{G}(t) e_{j}, v_{k}\right)_{\mathcal{H}}^{2} d t
\end{aligned}
$$


in $[0, T]$, for all $1 \leq k \leq m$ and all $m \geq 1$, and summing in $k$, we therefore obtain

$$
\begin{aligned}
& \left|\widetilde{u}_{m}(t)-u_{m}(t)\right|_{\mathcal{H}}^{2}+2 \int_{0}^{t}\left\langle\widetilde{A}\left(\widetilde{u}_{m}(s)-u_{m}(s)\right), \widetilde{u}_{m}(s)-u_{m}(s)\right\rangle d s \\
& +2 \int_{0}^{t}\left\langle\mathcal{B}(s)-\widetilde{B}\left(u_{m}(s), u_{m}(s)\right), \widetilde{u}_{m}(s)-u_{m}(s)\right\rangle d s \\
& =2 \int_{0}^{t}\left\langle\Phi(s)-\widetilde{F}\left(s, u_{m}(s)\right), \widetilde{u}_{m}(s)-u_{m}(s)\right\rangle d s \\
& +2 \sum_{j=1}^{m} \int_{0}^{t}\left(\mathcal{G}(s) e_{j}-\widetilde{G}\left(s, u_{m}(s)\right) e_{j}, \widetilde{u}_{m}(s)-u_{m}(s)\right)_{\mathcal{H}} d \beta_{s}^{j} \\
& +2 \sum_{j=m+1}^{\infty} \int_{0}^{t}\left(\mathcal{G}(s) e_{j}, \widetilde{u}_{m}(s)-u_{m}(s)\right)_{\mathcal{H}} d \beta_{s}^{j} \\
& +\sum_{j=1}^{m} \int_{0}^{t}\left|\Pi_{m}\left(\mathcal{G}(s) e_{j}-\widetilde{G}\left(s, u_{m}(s)\right) e_{j}\right)\right|_{\mathcal{H}}^{2} d s+\sum_{j=m+1}^{\infty} \int_{0}^{t}\left|\Pi_{m}\left(\mathcal{G}(s) e_{j}\right)\right|_{\mathcal{H}}^{2} d s .
\end{aligned}
$$

Denote now $\rho(t)=\exp \left(-\eta_{1} t-\eta_{2} \int_{0}^{t}\|u(s)\|_{\mathcal{U}}^{2} d s\right), \quad 0 \leq t \leq T$, with $\eta_{1}$ and $\eta_{2}$ positive constants to be fixed later. Applying Itô's formula to the process $\rho(t)\left|\widetilde{u}_{m}(t)-u_{m}(t)\right|_{\mathcal{H}}^{2}$, we obtain from (4.25), a2), and the properties of $\widetilde{B}, \widetilde{F}$ and $\Pi_{m}$,

$$
\begin{aligned}
& \rho(t)\left|\widetilde{u}_{m}(t)-u_{m}(t)\right|_{\mathcal{H}}^{2}+\widetilde{\alpha} \int_{0}^{t} \rho(s)\left\|\widetilde{u}_{m}(s)-u_{m}(s)\right\|_{\mathcal{U}}^{2} d s \\
& \leq 2 \int_{0}^{t} \rho(s)\left\langle\widetilde{B}\left(\widetilde{u}_{m}(s), \widetilde{u}_{m}(s)\right)-\mathcal{B}(s), \widetilde{u}_{m}(s)-u_{m}(s)\right\rangle d s \\
& +2 \widetilde{c} \mu \int_{0}^{t} \rho(s)\|u(s)\|_{\mathcal{U}}\left\|\widetilde{u}_{m}(s)-u_{m}(s)\right\|_{\mathcal{U}}\left|\widetilde{u}_{m}(s)-u_{m}(s)\right|_{\mathcal{H}} d s \\
& +2 \int_{0}^{t} \rho(s)\left\langle\Phi(s)-\widetilde{F}\left(s, \widetilde{u}_{m}(s)\right), \widetilde{u}_{m}(s)-u_{m}(s)\right\rangle d s \\
& +2 L_{\widetilde{F}} \int_{0}^{t} \rho(s)\left\|\widetilde{u}_{m}(s)-u_{m}(s)\right\|_{\mathcal{U}}\left|\widetilde{u}_{m}(s)-u_{m}(s)\right|_{\mathcal{H}} d s \\
& +2 \sum_{j=1}^{m} \int_{0}^{t} \rho(s)\left(\mathcal{G}(s) e_{j}-\widetilde{G}\left(s, u_{m}(s)\right) e_{j}, \widetilde{u}_{m}(s)-u_{m}(s)\right)_{\mathcal{H}} d \beta_{s}^{j} \\
& +2 \sum_{j=m+1}^{\infty} \int_{0}^{t} \rho(s)\left(\mathcal{G}(s) e_{j}, \widetilde{u}_{m}(s)-u_{m}(s)\right)_{\mathcal{H}} d \beta_{s}^{j} \\
& +\int_{0}^{t} \rho(s)\left\|\mathcal{G}(s)-\widetilde{G}\left(s, u_{m}(s)\right)\right\|_{\mathcal{L}^{2}(K ; \mathcal{H})}^{2} d s+\sum_{j=m+1}^{\infty} \int_{0}^{t} \rho(s)\left|\mathcal{G}(s) e_{j}\right|_{\mathcal{H}}^{2} d s \\
& -\eta_{1} \int_{0}^{t} \rho(s)\left|\widetilde{u}_{m}(s)-u_{m}(s)\right|_{\mathcal{H}}^{2} d s-\eta_{2} \int_{0}^{t} \rho(s)\|u(s)\|_{\mathcal{U}}^{2}\left|\widetilde{u}_{m}(s)-u_{m}(s)\right|_{\mathcal{H}}^{2} d s,
\end{aligned}
$$

for all $t \in[0, T]$ and all $m \geq 1$.

Article submitted to Royal Society 
Now, observe that

$$
\begin{aligned}
& \left\|\mathcal{G}(s)-\widetilde{G}\left(s, u_{m}(s)\right)\right\|_{\mathcal{L}^{2}(K ; \mathcal{H})}^{2}=\left\|\widetilde{G}(s, u(s))-\widetilde{G}\left(s, u_{m}(s)\right)\right\|_{\mathcal{L}^{2}(K ; \mathcal{H})}^{2} \\
& +2\left(\left(\mathcal{G}(s)-\widetilde{G}\left(s, u_{m}(s)\right), \mathcal{G}(s)-\widetilde{G}(s, u(s))\right)\right)_{\mathcal{L}^{2}(K ; \mathcal{H})} \\
& -\|\widetilde{G}(s, u(s))-\mathcal{G}(s)\|_{\mathcal{L}^{2}(K ; \mathcal{H})}^{2} \\
& \leq 2 L_{\widetilde{G}}^{2}\left|u(s)-\widetilde{u}_{m}(s)\right|_{\mathcal{H}}^{2}+2 L_{\widetilde{G}}^{2}\left|\widetilde{u}_{m}(s)-u_{m}(s)\right|_{\mathcal{H}}^{2} \\
& +2\left(\left(\mathcal{G}(s)-\widetilde{G}\left(s, u_{m}(s)\right), \mathcal{G}(s)-\widetilde{G}(s, u(s))\right)\right)_{\mathcal{L}^{2}(K ; \mathcal{H})} \\
& -\|\widetilde{G}(s, u(s))-\mathcal{G}(s)\|_{\mathcal{L}^{2}(K ; \mathcal{H})}^{2}
\end{aligned}
$$

On the other hand,

$$
\begin{aligned}
& 2 \widetilde{c} \mu\|u(s)\|_{\mathcal{U}}\left\|\widetilde{u}_{m}(s)-u_{m}(s)\right\|_{\mathcal{U}}\left|\widetilde{u}_{m}(s)-u_{m}(s)\right|_{\mathcal{H}} \\
& \leq \frac{\widetilde{\alpha}}{4}\left\|\widetilde{u}_{m}(s)-u_{m}(s)\right\|_{\mathcal{U}}^{2}+\frac{4 \widetilde{c}^{2} \mu^{2}}{\widetilde{\alpha}}\|u(s)\|_{\mathcal{U}}^{2}\left|\widetilde{u}_{m}(s)-u_{m}(s)\right|_{\mathcal{H}}^{2},
\end{aligned}
$$

and

$$
\begin{aligned}
& 2 L_{\widetilde{F}}\left\|\widetilde{u}_{m}(s)-u_{m}(s)\right\|_{\mathcal{U}}\left|\widetilde{u}_{m}(s)-u_{m}(s)\right|_{\mathcal{H}} \\
& \leq \frac{\widetilde{\alpha}}{4}\left\|\widetilde{u}_{m}(s)-u_{m}(s)\right\|_{\mathcal{U}}^{2}+\frac{4 L_{\widetilde{F}}^{2}}{\widetilde{\alpha}}\left|\widetilde{u}_{m}(s)-u_{m}(s)\right|_{\mathcal{H}}^{2} .
\end{aligned}
$$

On account of (4.27), (4.28) and (4.29), we obtain from (4.26), for $t \in[0, T], m \geq 1$,

$$
\begin{aligned}
& \rho(t)\left|\widetilde{u}_{m}(t)-u_{m}(t)\right|_{\mathcal{H}}^{2}+\frac{\widetilde{\alpha}}{2} \int_{0}^{t} \rho(s)\left\|\widetilde{u}_{m}(s)-u_{m}(s)\right\|_{\mathcal{U}}^{2} d s \\
& +\int_{0}^{t} \rho(s)\|\widetilde{G}(s, u(s))-\mathcal{G}(s)\|_{\mathcal{L}^{2}(K ; \mathcal{H})}^{2} d s \\
& \leq\left(\frac{4 L_{\widetilde{F}}^{2}}{\widetilde{\alpha}}+2 L_{\widetilde{G}}^{2}-\eta_{1}\right) \int_{0}^{t} \rho(s)\left|\widetilde{u}_{m}(s)-u_{m}(s)\right|_{\mathcal{H}}^{2} d s \\
& +\left(\frac{4 \widetilde{c}^{2} \mu^{2}}{\widetilde{\alpha}}-\eta_{2}\right) \int_{0}^{t} \rho(s)\|u(s)\|_{\mathcal{U}}^{2}\left|\widetilde{u}_{m}(s)-u_{m}(s)\right|_{\mathcal{H}}^{2} d s \\
& +2 \int_{0}^{t} \rho(s)\left\langle\widetilde{B}\left(\widetilde{u}_{m}(s), \widetilde{u}_{m}(s)\right)-\mathcal{B}(s), \widetilde{u}_{m}(s)-u_{m}(s)\right\rangle d s \\
& +2 \int_{0}^{t} \rho(s)\left\langle\Phi(s)-\widetilde{F}\left(s, \widetilde{u}_{m}(s)\right), \widetilde{u}_{m}(s)-u_{m}(s)\right\rangle d s \\
& +\sum_{j=m+1}^{\infty} \int_{0}^{t} \rho(s)\left|\mathcal{G}(s) e_{j}\right|_{\mathcal{H}}^{2} d s+2 L_{\widetilde{G}}^{2} \int_{0}^{t} \rho(s)\left|u(s)-\widetilde{u}_{m}(s)\right|_{\mathcal{H}}^{2} d s \\
& \left.+2 \int_{0}^{t} \rho(s)\left(\left(\mathcal{G}(s)-\widetilde{G}\left(s, u_{m}(s)\right), \mathcal{G}(s)-\widetilde{G}_{(s, u}(s)\right)\right)\right)_{\mathcal{L}^{2}(K ; \mathcal{H})} d s \\
& +2 \sum_{j=1}^{m} \int_{0}^{t} \rho(s)\left(\mathcal{G}(s) e_{j}-\widetilde{G}\left(s, u_{m}(s)\right) e_{j}, \widetilde{u}_{m}(s)-u_{m}(s)\right)_{\mathcal{H}} d \beta_{s}^{j} \\
& +\sum_{j=m+1}^{\infty} \int_{0}^{t} \rho(s)\left(\mathcal{G}(s) e_{j}, \widetilde{u}_{m}(s)-u_{m}(s)\right)_{\mathcal{H}} d \beta_{s}^{j} .
\end{aligned}
$$


Therefore, if we take $\eta_{1}=4 L_{\widetilde{F}}^{2} / \widetilde{\alpha}+2 L_{\widetilde{G}}^{2}, \quad \eta_{2}=4 \widetilde{c}^{2} \mu^{2} / \widetilde{\alpha}$, and for each integer $n \geq 1$ consider the $\mathcal{F}_{t}$-stopping time $\tau_{n}$ defined by

$$
\tau_{n}=\min \left(T, \inf \left\{t \in[0, T] ;|u(t)|_{\mathcal{H}}^{2}+\int_{0}^{t}\|u(s)\|_{\mathcal{U}}^{2} d s \geq n^{2}\right\}\right)
$$

it is straightforward to obtain from (4.30) and for all $n, m \geq 1$ that

$$
\begin{aligned}
& E\left(\rho\left(\tau_{n}\right)\left|\widetilde{u}_{m}\left(\tau_{n}\right)-u_{m}\left(\tau_{n}\right)\right|_{\mathcal{H}}^{2}\right)+\frac{\widetilde{\alpha}}{2} E \int_{0}^{\tau_{n}} \rho(s)\left\|\widetilde{u}_{m}(s)-u_{m}(s)\right\|_{\mathcal{U}}^{2} d s \\
& +E \int_{0}^{\tau_{n}} \rho(s)\|\widetilde{G}(s, u(s))-\mathcal{G}(s)\|_{\mathcal{L}^{2}(K ; \mathcal{H})}^{2} d s \\
& \leq \sum_{j=m+1}^{\infty} E \int_{0}^{T} \rho(s)\left|\mathcal{G}(s) e_{j}\right|_{\mathcal{H}}^{2} d s+2 L_{\widetilde{G}}^{2} E \int_{0}^{T} \rho(s)\left|u(s)-\widetilde{u}_{m}(s)\right|_{\mathcal{H}}^{2} d s \\
& +2 E \int_{0}^{\tau_{n}} \rho(s)\left(\left(\mathcal{G}(s)-\widetilde{G}\left(s, u_{m}(s)\right), \mathcal{G}(s)-\widetilde{G}(s, u(s))\right)\right)_{\mathcal{L}^{2}(K ; \mathcal{H})} d s \\
& +2 E \int_{0}^{\tau_{n}} \rho(s)\left\langle\widetilde{B}\left(\widetilde{u}_{m}(s), \widetilde{u}_{m}(s)\right)-\mathcal{B}(s)+\Phi(s)-\widetilde{F}\left(s, \widetilde{u}_{m}(s)\right), \widetilde{u}_{m}(s)-u_{m}(s)\right\rangle d s,
\end{aligned}
$$

Now, it follows that

$$
\lim _{m \rightarrow \infty}\left(\sum_{j=m+1}^{\infty} E \int_{0}^{T} \rho(s)\left|\mathcal{G}(s) e_{j}\right|_{\mathcal{H}}^{2} d s+2 L_{\widetilde{G}}^{2} E \int_{0}^{T} \rho(s)\left|u(s)-\widetilde{u}_{m}(s)\right|_{\mathcal{H}}^{2} d s\right)=0 .
$$

Also, as $\widetilde{G}\left(t, u_{m}\right) \rightarrow \mathcal{G}(t)$ in $M_{\mathcal{F}_{t}}^{2}\left(0, T ; \mathcal{L}^{2}(K ; \mathcal{H})\right)$, and $1_{\left[0, \tau_{n}\right]} \rho(t)(\mathcal{G}-\widetilde{G}(t, u(t))) \in$ $M_{\mathcal{F}_{t}}^{2}\left(0, T ; \mathcal{L}^{2}(K ; \mathcal{H})\right)$, we have

$$
\lim _{m \rightarrow \infty} E \int_{0}^{\tau_{n}} \rho(s)\left(\left(\mathcal{G}(s)-\widetilde{G}\left(s, u_{m}(s)\right), \mathcal{G}(s)-\widetilde{G}(s, u(s))\right)\right)_{\mathcal{L}^{2}(K ; \mathcal{H})} d s=0 .
$$

On the other hand,

$$
\begin{aligned}
& E \int_{0}^{\tau_{n}} \rho(s)\left\langle\widetilde{B}\left(\widetilde{u}_{m}(s), \widetilde{u}_{m}(s)\right)-\mathcal{B}(s)+\Phi(s)-\widetilde{F}\left(s, \widetilde{u}_{m}(s)\right), \widetilde{u}_{m}(s)-u_{m}(s)\right\rangle d s \\
& =E \int_{0}^{\tau_{n}} \rho(s)\left\langle\widetilde{B}\left(\widetilde{u}_{m}(s), \widetilde{u}_{m}(s)\right)-\widetilde{B}(u(s), u(s)), \widetilde{u}_{m}(s)-u_{m}(s)\right\rangle d s \\
& +E \int_{0}^{\tau_{n}} \rho(s)\left\langle\widetilde{F}(s, u(s))-\widetilde{F}\left(s, \widetilde{u}_{m}(s)\right), \widetilde{u}_{m}(s)-u_{m}(s)\right\rangle d s \\
& +E \int_{0}^{\tau_{n}} \rho(s)\left\langle\Phi(s)-\widetilde{F}(s, u(s))+\widetilde{B}(u(s), u(s))-\mathcal{B}(s), \widetilde{u}_{m}(s)-u_{m}(s)\right\rangle d s
\end{aligned}
$$

But, thanks to (4.17) and (4.24), $\widetilde{u}_{m}-u_{m} \rightarrow 0$ in $M_{\mathcal{F}_{t}}^{2}(0, T ; \mathcal{U})$, as $m \rightarrow \infty$, and it is also immediate that

$$
1_{\left[0, \tau_{n}\right]} \rho(t)(\Phi(t)-\widetilde{F}(t, u(t))+\widetilde{B}(u(t), u(t))-\mathcal{B}(t)) \in M_{\mathcal{F}_{t}}^{2}\left(0, T ; \mathcal{U}^{*}\right),
$$


and $1_{\left[0, \tau_{n}\right]} \rho(t) \widetilde{F}(t, u(t))-\widetilde{F}\left(t, \widetilde{u}_{m}(t)\right) \rightarrow 0$ in $M_{\mathcal{F}_{t}}^{2}\left(0, T ; \mathcal{U}^{*}\right)$, as $m \rightarrow \infty$, what implies

$$
\lim _{m \rightarrow \infty} E \int_{0}^{\tau_{n}} \rho(s)\left\langle\Phi(s)-\widetilde{F}(s, u(s))+\widetilde{B}(u(s), u(s))-\mathcal{B}(s), \widetilde{u}_{m}(s)-u_{m}(s)\right\rangle d s=0,
$$

and

$$
\lim _{m \rightarrow \infty} E \int_{0}^{\tau_{n}} \rho(s)\left\langle\widetilde{F}(s, u(s))-\widetilde{F}\left(s, \widetilde{u}_{m}(s)\right), \widetilde{u}_{m}(s)-u_{m}(s)\right\rangle d s=0 .
$$

Finally, by b2) and (4.23),

$$
\begin{aligned}
& \left\|1_{\left[0, \tau_{n}\right]} \rho(t)\left(\widetilde{B}\left(\widetilde{u}_{m}(t), \widetilde{u}_{m}(t)\right)-\widetilde{B}(u(t), u(t))\right)\right\|_{\mathcal{U}^{*}} \\
& \leq 1_{\left[0, \tau_{n}\right]}(t) \widetilde{c}\left(\left|\widetilde{u}_{m}(t)-u(t)\right|_{\mathcal{H}} \mu\|u(t)\|_{\mathcal{U}}+|u(t)|_{\mathcal{H}}\left\|\widetilde{u}_{m}(t)-u(t)\right\|_{\mathcal{U}}\right),
\end{aligned}
$$

and thus $\left\|1_{\left[0, \tau_{n}\right]} \rho(t)\left(\widetilde{B}\left(\widetilde{u}_{m}(t), \widetilde{u}_{m}(t)\right)-\widetilde{B}(u(t), u(t))\right)\right\|_{\mathcal{U}^{*}} \rightarrow 0$, as $m \rightarrow \infty, d t \times d P-$ a.e., and

$\left\|1_{\left[0, \tau_{n}\right]} \rho(t)\left(\widetilde{B}\left(\widetilde{u}_{m}(t), \widetilde{u}_{m}(t)\right)-\widetilde{B}(u(t), u(t))\right)\right\|_{\mathcal{U}^{*}} \leq(3 \mu+1) n \widetilde{c}\|u(t)\|_{\mathcal{U}} \in M_{\mathcal{F}_{t}}^{2}(0, T ; \mathbb{R})$,

whence

$$
\lim _{m \rightarrow \infty} E \int_{0}^{\tau_{n}} \rho(s)\left\langle\widetilde{B}\left(\widetilde{u}_{m}(s), \widetilde{u}_{m}(s)\right)-\widetilde{B}(u(s), u(s)), \widetilde{u}_{m}(s)-u_{m}(s)\right\rangle d s=0 .
$$

From (4.33) - (4.38), and the fact that $\exp \left(-\eta_{1} T-\eta_{2} n\right) \leq 1_{\left[0, \tau_{n}\right]} \rho(t) \leq 1$, we obtain from (4.32) and for all $n \geq 1$,

$$
\begin{gathered}
\lim _{m \rightarrow \infty} E\left(\left|\widetilde{u}_{m}\left(\tau_{n}\right)-u_{m}\left(\tau_{n}\right)\right|_{\mathcal{H}}^{2}\right)=0, \\
\lim _{m \rightarrow \infty} E \int_{0}^{\tau_{n}}\left\|\widetilde{u}_{m}(s)-u_{m}(s)\right\|_{\mathcal{U}}^{2} d s=0, \\
E \int_{0}^{\tau_{n}}\|\widetilde{G}(s, u(s))-\mathcal{G}(s)\|_{\mathcal{L}^{2}(K ; \mathcal{H})}^{2} d s=0,
\end{gathered}
$$

It is now clear that (4.41) and the fact that the sequence $\left\{\tau_{n} ; n \geq 1\right\}$ is increasing to $T$, imply that $\widetilde{G}(t, u(t))=\mathcal{G}(t)$, as elements of the space $M_{\mathcal{F}_{t}}^{2}\left(0, T ; \mathcal{L}^{2}(K ; \mathcal{H})\right)$. Also, observe that (4.40) and (4.24) imply

$$
u_{m} 1_{\left[0, \tau_{n}\right]} \rightarrow u 1_{\left[0, \tau_{n}\right]}, \quad \text { in } M_{\mathcal{F}_{t}}^{2}(0, T ; \mathcal{U}) .
$$

Thus, given any $w \in M_{\mathcal{F}_{t}}^{\infty}(0, T ; \mathcal{U})$,

$$
\begin{aligned}
& E \int_{0}^{\tau_{n}}\left\langle\widetilde{B}(u(s), u(s))-\widetilde{B}\left(u_{m}(s), u_{m}(s)\right), w(s)\right\rangle d s \\
& \leq \widetilde{c}\|w\|_{M_{\mathcal{F}_{\mathcal{H}}}^{\infty}(0, T ; \mathcal{U})} E \int_{0}^{\tau_{n}}\left(\left|u(s)-u_{m}(s)\right|_{\mathcal{H}} \mu\|u(s)\|_{\mathcal{U}}+\left|u_{m}(s)\right|_{\mathcal{H}}\left\|u(s)-u_{m}(s)\right\|_{\mathcal{U}}\right) d s,
\end{aligned}
$$


and consequently, by (4.42),

$$
\lim _{m \rightarrow \infty} E \int_{0}^{\tau_{n}}\left\langle\widetilde{B}(u(s), u(s))-\widetilde{B}\left(u_{m}(s), u_{m}(s)\right), w(s)\right\rangle d s=0 .
$$

Taking into account (4.19), it follows from (4.43) that

$$
E \int_{0}^{\tau_{n}}\langle\widetilde{B}(u(s), u(s))-\mathcal{B}(s), w(s)\rangle d s=0, \forall w \in M_{\mathcal{F}_{t}}^{\infty}(0, T ; \mathcal{U}),
$$

and consequently, as $\tau_{n} \uparrow T$ and $M_{\mathcal{F}_{t}}^{\infty}(0, T ; \mathcal{U})$ is dense in $M_{\mathcal{F}_{t}}^{2}(0, T ; \mathcal{U})$, we obtain from (4.44) that $\widetilde{B}(u(t), u(t))=\mathcal{B}(t)$, as elements of the space $M_{\mathcal{F}_{t}}^{2}\left(0, T ; \mathcal{U}^{*}\right)$.

Analogously, one can prove that $\widetilde{F}(t, u(t))=\Phi(t)$, as elements of $M_{\mathcal{F}_{t}}^{2}\left(0, T ; \mathcal{U}^{*}\right)$, and thus, $u$ is the solution of (4.3).

Step 5.- Obtention of the estimate (4.9).

Using the sequence of stopping times $\tau_{n}$ defined by (4.31), and arguing as for the obtention of (4.15), one obtains (4.9).

It is not difficult to prove the following result (see Breckner 1999).

Lemma 4.5. Let $\left\{Q_{m} ; m \geq 1\right\} \subset M_{\mathcal{F}_{t}}^{2}(0, T ; \mathbb{R})$ be a sequence of continuous real processes, and let $\left\{\sigma_{n} ; n \geq 1\right\}$ be a sequence of $\mathcal{F}_{t}$-stopping times such that $\sigma_{n} \uparrow T$, $\sup _{m \geq 1} E\left|Q_{m}(T)\right|^{2}<\infty$, and $\lim _{m \rightarrow \infty} E\left|Q_{m}\left(\sigma_{n}\right)\right|=0$, for all $n \geq 1$. Then $\lim _{m \rightarrow \infty} E\left|Q_{m}(T)\right|=0$.

Applying this Lemma to $Q_{m}(t)=\left|u(t)-u_{m}(t)\right|_{\mathcal{H}}^{2}$ and $\sigma_{n}=\tau_{n}$, and taking into account (4.9), (4.15), (4.39) and the uniqueness of $u$, one easily obtains that the whole sequence $u_{m}$ defined by (4.10) satisfies $\lim _{m \rightarrow \infty} E\left|u(t)-u_{m}(t)\right|_{\mathcal{H}}^{2}=0$, for all $t \in[0, T]$.

Analogously, applying the Lemma to $Q_{m}(t)=\int_{0}^{t}\left\|u(s)-u_{m}(s)\right\|_{\mathcal{U}}^{2} d s$ and $\sigma_{n}=$ $\tau_{n}$, and taking into account (4.9), (4.15), (4.40) and the uniqueness of $u$, we have that the whole sequence $u_{m}$ defined by $(4.10)$ converges to $u$ strongly in $M_{\mathcal{F}_{t}}^{2}(0, T ; \mathcal{U})$, i.e. it satisfies $\lim _{m \rightarrow \infty} E \int_{0}^{t}\left\|u(s)-u_{m}(s)\right\|_{\mathcal{U}}^{2} d s=0$.

\section{Proof of Proposition 3.2 and Theorem 3.3}

As we have already mentioned, the results stated in Proposition 3.2 and Theorem 3.3 are direct consequences of, respectively, Proposition 4.2 and Proposition 4.3, and Theorem 4.4, which will be proved in this section.

To this end, we take: $\mathcal{H}=V$, with $(u, v)_{\mathcal{H}}=((u, v))$, and $\mathcal{U}=D(A)$, with $((u, v))_{\mathcal{U}}=(A u, A v)$. We define $\langle\widetilde{A} u, v\rangle=\nu(A u, v)+\nu \alpha(A u, A v)$, for $u, v \in D(A)$, and observe that $\widetilde{A}$ satisfies a1), a2) with $\widetilde{\alpha}=2 \nu \alpha$, and (4.2) is fulfilled with $\lambda_{k}=\nu \mu_{k}, v_{k}=w_{k} / \sqrt{1+\alpha \mu_{k}}$, where $\mu_{k}$ and $w_{k}$ are the eigenvalues of $A$ and their corresponding associated eigenvectors.

On the other hand, we consider $\widetilde{B}(u, v)$ and $\widetilde{F}(t, u)$ defined by

$$
\begin{gathered}
\langle\widetilde{B}(u, v), w\rangle=b^{\#}(u, v-\alpha \Delta v, w), \quad \forall(u, v, w) \in D(A) \times D(A) \times D(A), \\
\langle\widetilde{F}(t, u), w\rangle=\langle F(t, u), w\rangle, \quad \forall(u, w) \in V \times D(A) .
\end{gathered}
$$

Article submitted to Royal Society 
Finally, let $\widetilde{G}(t, u)$ be defined by

$$
\widetilde{G}(t, u)=(I+\alpha A)^{-1} \circ \mathcal{P} \circ G(t, u), \quad \forall(t, u) \in(0, T) \times V,
$$

where $I$ denotes the identity operator in $H$. First of all, observe that the operator $I+\alpha A$ is bijective from $D(A)$ onto $H$, and

$$
\left(\left((I+\alpha A)^{-1} h, w\right)\right)=(h, w), \quad \forall h \in H, \quad \forall w \in V .
$$

Thus, in particular, $\left\|(I+\alpha A)^{-1} h\right\|^{2} \leq \frac{1}{1+\alpha \mu_{1}}|h|^{2}$, for all $h \in H$. Also, observe that for each $j \geq 1,\left(G(t, u) e_{j}, w\right)=\left((I+\alpha A)\left(\widetilde{G}(t, u) e_{j}\right), w\right)=\left(\left(\widetilde{G}(t, u) e_{j}, w\right)\right)$, for all $(t, u, w) \in(0, T) \times V \times D(A)$, and consequently, for all $(t, w) \in[0, T] \times D(A)$,

$$
\left(\int_{0}^{t} G(s, u(s)) d W_{s}, w\right)=\sum_{j=1}^{\infty} \int_{0}^{t}\left(G(s, u(s)) e_{j}, w\right) d \beta_{s}^{j}=\left(\left(\int_{0}^{t} \widetilde{G}(s, u(s)) d W_{s}, w\right)\right) .
$$

By means of a straightforward application of Propositions 4.2 and 4.3 , and Theorem 4.4, we deduce Proposition 3.2 and the existence of $u$ in Theorem 3.3.

For the existence of the pressure $p$, observe that by (2.3) and (2.5), as $u \in$ $L^{4}\left(\Omega ; L^{2}(0, T ; D(A))\right)$ and is $\mathcal{F}_{t}$-progressively measurable, then

$$
(u \cdot \nabla)(u-\alpha \Delta u)+\nabla u^{*} \cdot \Delta u \in L^{2}\left(\Omega, \mathcal{F}_{t}, P ; L^{1}\left(0, t ;\left(H^{-1}(D)\right)^{3}\right)\right),
$$

with

$$
\begin{gathered}
E\left[\left(\int_{0}^{t}\left\|(u(s) \cdot \nabla)(u(s)-\alpha \Delta u(s))+\nabla u(s)^{*} \cdot \Delta u(s)\right\|_{\left(H^{-1}(D)\right)^{3}} d s\right)^{2}\right] \\
\leq c E\left[\left(\int_{0}^{t}|A u(s)|^{2} d s\right)^{2}\right], \quad \forall t \in[0, T] .
\end{gathered}
$$

On the other hand, $u-\alpha \Delta u \in L^{4}\left(\Omega, \mathcal{F}_{t}, P ; L^{2}\left(0, t ;\left(L^{2}(D)\right)^{3}\right)\right)$, and consequently $\partial_{t}(u-\alpha \Delta u) \in L^{4}\left(\Omega, \mathcal{F}_{t}, P ; H^{-1}\left(0, t ;\left(L^{2}(D)\right)^{3}\right)\right)$, for all $t \in[0, T]$. Also, as $u \in$ $L^{4}(\Omega, \mathcal{F}, P ; C([0, T] ; V))$, and is $\mathcal{F}_{t}$-progressively measurable, then it follows that $F(t, u) \in L^{4}\left(\Omega, \mathcal{F}_{t}, P ; L^{2}\left(0, t ;\left(H^{-1}(D)\right)^{3}\right)\right)$, and reasoning as in Langa et al. (2003), one also obtains $G(t, u) \dot{W}_{t} \in L^{4}\left(\Omega, \mathcal{F}_{t}, P ; W^{-1, \infty}\left(0, t ;\left(L^{2}(D)^{3}\right)\right)\right)$, for all $t \in[0, T]$. Finally, for the term $A u-\alpha \Delta(A u)$, which is the more irregular one, as $u \in$ $L^{4}\left(\Omega ; L^{2}(0, T ; D(A))\right)$, we then have

$$
A u-\alpha \Delta(A u) \in L^{4}\left(\Omega, \mathcal{F}_{t}, P ; L^{2}\left(0, t ;\left(H^{-2}(D)\right)^{3}\right)\right), \forall t \in[0, T] .
$$

Arguing as in Langa et al. (2003), and in particular using Remark 4.3 in this paper, one can prove the existence and uniqueness of $p$.

We thank the referees for their interesting comments and suggestions. This work has been partially supported by MCYT (Ministerio de Ciencia y Tecnología (Spain)) and FEDER (Fondo Europeo de Desarrollo Regional) under the Project BFM2002-03068. 


\section{References}

Bensoussan, A., 1995 Stochastic Navier-Stokes equations, Acta Appl. Math. 38, no. 3, 267-304.

Bensoussan, A. \& Temam, R., 1973 Équations stochastiques du type Navier-Stokes, J. Functional Analysis 13, 195-222.

Breckner (Lisei), H., 1999 Approximation and optimal control of the stochastic NavierStokes equation, Dissertation, Martin-Luther University, Halle-Wittenberg.

Caraballo, T. \& Langa, J.A., 2001 Comparison of the long-time behaviour of linear Ito and Stratonovich partial differential equations, Stoch. Anal. Appl. 19 (2), 183-195.

Coutand, D., Peirce, J., \& Shkoller, S., 2002 Global well-posedness of weak solutions for the Lagrangian averaged Navier-Stokes equations on bounded domains, Comm. on Pure and Appl. Anal. 1, no.1, 35-50.

DaPrato, G., \& Zabczyk, J., 1992 Stochastic equations in infinite dimensions, Cambridge University Press, Cambridge, 1992.

Ecke, R., 2005 The turbulence problem. An experimentalist's perspective, Los Alamos Science 29, 124-141.

Foias, C., Holm, D.D., \& Titi, E.S., 2002 The three dimensional viscous Camassa-Holm equations and their relation to the Navier-Stokes equations and turbulence theory, $J$. Dyn. Diff. Eq. 14, no. 1, 1-35.

Holm, D.D., Jeffery, C., Kurien, S., Livescu, D., Taylor, M.A., \& B.A. Wingate, 2005 The LANS- $\alpha$ model for computing turbulence, Los Alamos Science 29, 152-171.

Holm, D.D., 2005 Taylor's Hypothesis, Hamilton's Principle, and the LANS- $\alpha$ model for computing turbulence, Los Alamos Science 29, 172-180.

Holm, D. D., Putkaradze, V., Weidman, P D., Wingate, B. A., 2003 Boundary effects on exact solutions of the Lagrangian-averaged Navier-Stokes- $\alpha$ equations, Progress in statistical hydrodynamics (Santa Fe, NM, 2002), J. Statist. Phys. 113, no. 5-6, 841-854.

Langa, J.A., Real, J., \& Simon, J., (2003) Existence and regularity of the pressure for the stochastic Navier-Stokes equations, Appl. Math. Optim. 48, no. 3, 195-210.

Marsden, J.E. \& Shkoller, S., 2001 Global well-posedness for the LANS- $\alpha$ equations on bounded domains, R. Soc. Lond. Philos. Trans. Ser. A Math. Phys. Eng. Sci. 359, no. 1784, 1449-1468.

Mikulevicius, R. \& Rozovskii, B.L., 2004 Stochastic Navier-Stokes for turbulent flows, SIAM J. Math. Anal. 35 no. 5, 1250-1310.

Mikulevicius, R. \& Rozovskii, B.L., 2005 Global $L_{2}$-solutions of Stochastic Navier-Stokes equations, Ann. Prob. 33, no. 1, 137-176.

Pardoux, E., 1975 Équations aux Dérivées Partielles Stochastiques Non Linéaires Monotones, Thèse, Université Paris XI.

Simon, J., 1999 On the existence of the pressure for solutions of the variational NavierStokes equations, J. Math. Fluid Mech. 1, 225-234.

Article submitted to Royal Society 